ROZEMARIA KRYSTYNA TERTEL

\title{
Über einen Versuch der Adjustierung einer fehlerhaften Artikulation des Deutschen bei Studenten der Germanistik
}

$\mathrm{Zu}$ Beginn des akademischen Jahres 1962/63 erhielt die Verfasserin dieses Berichts vom Lehrstuhl für Germanistik der Warschauer Universität den Auftrag, mit einer Studentengruppe des ersten Studienjahres praktische Übungen der deutschen Sprache zu führen. Die Gruppe bestand aus zwölf Studenten, deren Alter verschieden war. Einer der Studenten war schon über dreißig Jahre alt, drei andere standen im Alter zwischen 21 und 26 Jahren, die übrigen waren jünger, davon manche noch nicht ganz 18. Für die Übungen waren acht Lektionsstunden wöchentlich vorgesehen, aber leider nur vier Zusammenkünfte, denn es mußten vor allem wegen Mangel an Übungssälen je zwei Stunden zusammengelegt werden. Bei der Auswahl des in den Übungen zur Verwendung kommenden Materials wurde von Seiten der Leitung des Lehrstuhls weitgehend freie Hand gelassen, dasselbe hinsichtlich der für derartige Übungen geeigneten Lehrmethode.

Mit dem oben genannten Auftrag wurde der Autorin dieses Berichts zum erstenmal die Möglichkeit gegeben, mit Studenten der Germanistik, die ihr bisher nur von der eigenen Studienzeit bekannt waren, zu arbeiten. Deshalb drängte sich unwillkürlich schon nach der ersten Begegnung mit der Studentengruppe der Eindruck auf, daß das Niveau der Sprachkenntnisse, oder, exakter ausgedrückt, der Sprechfähigkeiten der Studenten des ersten Studienjahres während der letzten zehn Jahre leider bedeutend gesunken ist. Früher beherrschten mindestens $80 \%$ der das Studium beginnenden Studenten voll den Kode der deutschen Sprache, und die praktischen Übungen während der Studien waren nur dazu bestimmt, diese zum größten Teil nicht in der Schule erworbenen Sprachkenntnisse zu pflegen und zu vertiefen. Heute ist das Niveau der Sprechfähigkeiten der Studenten vielleicht ausgeglichener, aber leider in negativem Sinn. Von einem vollen Beherrschen des Kodes der deutschen Sprache kann keinesfalls die Rede sein, und der Begriff ,aus- 
geglichen" bezieht sich nur darauf, daß fast alle Studenten die gleichen ernsthaften Lücken aufweisen und dieselben Fehler machen. Die Studenten der besprochenen Gruppe hatten mit zwei Ausnahmen die deutsche Sprache in der Oberschule gelernt, sie stammten aus verschiedenen Gegenden Polens, machten aber dieselben Fehler, alle wiesen sie die gleichen, in großem Maße ungenügenden Fähigkeiten, sich der deutschen Sprache aktiv zu bedienen, auf.

Bei einer auch nur oberflächlichen Analyse der Sprachkenntnisse der Studenten der Gruppe konnte festgestellt werden, daß sie eine verhältnismäßig gute Kenntnis der traditionellen beschreibenden Grammatik der deutschen Sprache aufwiesen, d.h. sie konnten in den meisten Fällen die wichtigsten Regeln aus dem Gedächtnis in polnischer Sprache, und manche von ihnen sogar deutsch, hersagen. Sie beherrschten auch in gewissem Grad den analytischen Schreibkode der deutschen Sprache, d.h. sie konnten einen nicht besonders komplizierten Text lesen und verstehen. Schon schlechter beschaffen war es mit dem Beherrschen des analytischen Sprechkodes der deutschen Sprache, denn es kostete die Studenten einige Mühe, ein normal gesprochenes Deutsch zu verstehen, sogar wenn der Sprecher bemüht war, einfache Sätze zu gebrauchen und im Bereich des Grundwortschatzes der Lehrbücher für Oberschulen blieb. Von einem Beherrschen der beiden synthetischen Kode, d.i. vom Sprechen sowie vom Schreiben im Sinne von selbständigem Komponieren von Texten in deutscher Sprache konnte leider kaum die Rede sein. Beim Sprechen kann es, wie es in solchen Fällen üblich ist, nicht zur sprachlichen Synthese im Unterbewußtsein, sondern zu einem bewußten Zusammensammeln der zum Aufbau eines Satzes nötigen morphologischen und syntaktischen Strukturen kommen (sie waren als eingeprägte grammatische Regeln im Bewußtsein und nicht als durch Hören und Sprechen automatisierte Strukturen im Unterbewußtsein vorhanden), was mit großem Zeitverlust verbunden war und in der Regel zum künstlichen Herumstottern führte, oder es kam beim Sprechen einfach $\mathrm{zu}$ einer starken Interferenz der Strukturen der Muttersprache. Es seien hier beispielsweise nur einige von den bei den Studenten auftretenden Erscheinungen der Interferenz der morphologischen und syntaktischen Strukturen der polnischen Sprache herausgegriffen: Fehlerhafte Wortfolge im Satz, und zwar, weil im Polnischen die Wortfolge im Satz, wenn auch nicht ganz beliebig, so doch verhältnismäßig frei ist, es im Deutschen aber einen ganz bestimmten Satzrahmen gibt, der streng beachtet werden muß. Oder es kam z.B. zu Fehlern beim Gebrauch des Reflexivpronomens „sich", da es im Polnischen ungebeugt bleibt, im Deutschen aber bei verschiedenen Personen verschiedene Formen aufweist, usw. Derartige Beispiele könnten leicht vermehrt werden, aber es 
ist nicht der Zweck dieser Arbeit, auf Interferenzfälle dieser Art einzugehen.

Bei sämtlichen Studenten der besprochenen Gruppe kam es unter anderem zu einer äußerst starken Interferenz der muttersprachlichen Artikulationbasis bei der Aussprache des Deutschen. Diese Interferenz war dermaßen stark ausgeprägt, daß jede, nur für die deuts che Sprache typische Lautsubstanz in die ihr im polnischen System am nächsten stehende Lautsubstanz umtransponiert wurde und so zum Ausdruck kam. Ein derartiges Transponieren ist nun aber absolut unzulässig, und das muß wohl nicht erst besonders betont werden, denn es ist allgemein bekannt, daß dies nicht nur zu einer Allophonierung führt, sondern daß auch der Phonembestand der deutschen Sprache angegriffen und umgebaut wird, was nicht nur ein Verstehen des Gesprochenen verhindert, sondern auch zu ernsthaften Mißverständnissen führt. Es ist schließlich nicht egal, ob jemand die Zügel oder die Ziegel in die Hand nimmt, ob wir die Fremdsprache kennen oder können, ob von Stahl oder Stall die Rede war, usw. Außerdem wird die Unverständlichkeit des Gesprochenen dann noch durch die Interferenz anderer muttersprachlicher Strukturen des Ausdrucksplanes auf die deutsche Sprache erheblich gesteigert, was auch im besprochenen Falle eintrat. $\mathrm{Zu}$ alledem trat in unserem Fall die Tatsache, daß die Studenten nun schon jahrelang so gesprochen hatten und die erwähnte Transponierung bei ihnen zu einer eingeprägten, ständigen Gewohnheit geworden war. Angesichts dessen tauchte die Frage auf, ob es noch eine Möglichkeit gab, diese Interferenz zu beseitigen.

Das Einfachste wäre es natürlich gewesen, diese Frage erst gar nicht aufzuwerfen und so vorzugehen, wie es leider die meisten Lektoren tun. Sie führen in den praktischen Übungen keine besonderen Sprechübungen, um bei den Studenten eine wenigstens relativ reine phonetische Aussprache zu erzielen, sondern sie sind nur bemüht, durch verschiedenartige · Übungen ihre Geläufigkeit im Sprechen überhaupt zu erweitern. Allem Anschein nach wird damit gerechnet, die Studenten würden mit der Zeit unter dem Einfluß der relativ einwadfrei sprechenden Lehrer ohne besondere Hilfe zu einer richtigen Artikulationsbasis gelangen. Dieses Vorgehen führt aber $\mathrm{zu}$ keinem befriedigenden Resultat und kann auch zu keinem führen, da man von einem, vom sprachwissenschaftlichen Standpunkt aus falschen Grundsatz ausgeht. Wenn man nämlich das Prinzip des möglichen Transponierens der Lautsubstanz beim Erlernen einer Fremdsprache nicht in Betracht zieht, und die so zustande kommende Interferenz der Muttersprache nicht durch spezielle, mechanisierende Übungen beseitigt, kann man nur mit gelegentlichen Zufallsprodukten rechnen (z.B. können verhältnis- 
mäßig gute Ergebnisse bei Studenten mit großem Nachahmungsvermögen erzielt werden), was jedoch besonders für Studenten der Germanistik zu wenig ist und keinesfalls zufriedenstellen darf. Die Folgen einer Vernachlässigung in dieser Hinsicht sind so schwerwiegend, daß sie nicht bagatelisiert werden dürfen. Es bedarf sicher keiner besonderen Betonung, daß Absolventen einer Neuphilologischen Fakultät nicht $\mathrm{nur}$ als Wissenschaftler arbeiten (wo angeblich, wie oft der Standpunkt vertreten wird, eine gute theoretische Sprachkenntnis genügt), sondern in überwiegender Zahl als Lehrer in die Schule gehen. Sie vermitteln dort den Schülern ihre unvollständigen praktischen Sprachkenntnisse und dazu eine ansehnliche Portion Metasprache, diese Schüler werden Studenten, Lehrer ... und der Kreis schließt sich. Verwundert es dann noch, wenn ein Kandidat, der Germanistik studieren will, in der Aufnahmeprüfung sagt: „Sie sprechen mit deutschem Akzent, ich verstehe Sie nicht"!?

Manchmal wird auch noch eine andere Ansicht über das besprochene Problem vertreten, und zwar die, daß es bei Erwachsenen, mit falsch eingeprägter Artikulationsbasis, also auch bei Studenten, unmöglich sei, eine verhältnismäßig richtige Artikulation der Fremdsprache zu erzielen. Daß dies nicht zutrifft, konnte durch die Arbeit mit der genannten Studentengruppe bewiesen werden.

Da die Interferenz der muttersprachlichen Artikulationsbasis in dem von den Studenten der besagten Gruppe gesprochenen Deutsch dermaßen stark war, daß der Inhalt des Gesprochenen sogar für ein an starke Interferenz gewöhntes $\mathrm{Ohr}$ unverständlich war, unterlag es gleich nach der ersten Übungsstunde keinem Zweifel, daß hier unbedingt auf dem schnellsten Wege mit dem Umbau der Artikulationsbasis begonnen werden mußte. Es durfte vor allem auf keinen Fall dazu kommen, daß ein weiteres Einprägen grundsätzlich falscher Formen zugelassen wurde. Später sollte es sich dann erweisen, daß damit ein Versuch unternommen wurde, dessen Gelingen bisher stark bezweifelt wurde — der Versuch der Adjustierung einer schon weitgehend eingeprägten fehlerhaften Artikulation bei Erwachsenen.

Das Aneignen einer fremdsprachlichen Artikulationsbasis durch Erwachsene ist eine keineswegs leichte Sache und kann nur durch mühevolle Arbeit zustande kommen. Eine noch viel schwierigere Sache ist es aber, eine fehlerhaft eingeprägte Artikulation, d. h. eine Artikulation mit weitgehender muttersprachlicher Interferenz $\mathrm{zu}$ adjustieren. Es darf erstens nicht damit gerechnet werden, daß eine eingehende Beschreibung der richtigen Artikulation genüge, um $\mathrm{zu}$ einem befriedigenden Resultat $\mathrm{zu}$ gelangen. Auch wenn man dieser Beschreibung noch genaue Hinweise auf die Unterschiede zwischen den 
Lautwerten der Muttersprache und der Fremdsprache hinzufügt, ist kein Erfolg zu erwarten. Jedes Lehrbuch der deutschen Sprache bringt z.B. diesbezügliche Beschreibungen und Hinweise, aber die daraus Lernenden sprechen meist mit starker Interferenz der Artikulation der Muttersprache. Nur systematisch und mit viel Fleiß (von Seiten der Schüler und des Lehrers) betriebene praktische Übungen können hier Abhilfe schaffen.

Im besprochenen Fall wurde die Arbeit an der Adjustierung mit einer für praktische Übungen zweckmäßigen Zusammenstellung der wichtigsten Unterschiede in der polnischen und deutschen Artikulation begonnen, damit ein systematisches Vorgehen und eine Steigerung der Schwierigkeiten bei der Beseitigung der einzelnen Interferenzfälle möglich war. Es erwies sich dabei, daß es nicht leicht ist, in der Fülle der interferierenden Fälle den Punkt zu finden, wo mit der Adjustierung $\mathrm{zu}$ beginnen ist. Im beschriebenen Fall wurden diesbezüglich einige Fehler gemacht, und erst die weitere Praxis in Anlehnung an die entsprechenden wissenschaftlichen Erkenntnisse wird es erlauben, die günstigste Reihenfolge bei der Beseitigung von fremden Erscheinungen in der Artikulation zu ermitteln. Einerseits muß alles so schnell wie möglich korrigiert werden, damit es nicht zu einer weiteren Einprägung der Fehler kommt, die ohnehin schon hartnäckig genug sind, aber alles auf einmal anzugreifen ist anderseits ein Ding der Unmöglichkeit. Im beschriebenen Fall wurde für die langen und kurzen Vokale entschieden, deren Aussprache nicht leicht fällt, da sie sich stark sowohl qualitativ als auch quantitativ von den polnischen Vokallauten unterscheiden.

Während der ersten zwei Monate wurden bei jeder Zusammenkunft mit den Studenten, d.i. viermal wöchentlich 10-15 Minuten rein phonetischen Übungen gewidmet, später wurden besondere phonetische Übungen nur noch dort eingeschoben, wo es sich erwiesen hatte, daß die Bildung gewisser Laute immer noch Schwierigkeiten schaffte (es waren meist Übungen für einzelne Studenten), sonst diente jeder normal in den praktischen Übungen zur Anwendung kommende Text, ja jeder während der Übungen ausgesprochene Satz gleichzeitig als phonetische Übung.

Bevor eine eingehende Beschreibung des Vorgangs der durchgeführten phonetischen Übungen, die eine Adjustierung der Artikulation zum Ziel hatten, erfolgt, muß noch ein Zufall erwähnt werden, der ein sonst unlogisch erscheinendes, methodisches Vorgehen erklärt, und der zu gewissen Erkenntnissen geführt hat.

Die Leitung des Lehrstuhls für Germanistik hatte $\mathrm{zu}$ Anfang des Studienjahres besonders betont, in den praktischen Übungen seien kei- 
nerlei Probleme der beschreibenden Grammatik theoretisch zu behandeln, damit nicht dubliert werde, was in dafür vorgesehenen Vorlesungen und Übungen gesagt werde. Weil nun gewöhnlich die Vorlesungen und Seminare über deskriptive Grammatik mit dem phonetischen Teil beginnen, wurde auch in diesem Fall angenommen, daß die theoretische Erklärung der Artikulationsbasis der einzelnen deutschen Laute schon in den ersten Vorlesungen erfolge, und aus diesem Grunde wurden die Laute ohne jede Einführung sofort rein praktisch geübt. Es erwies sich dann erst einige Zeit später, daß damit auf etwas gebaut wurde, das noch gar nicht da war, denn die besagten Vorlesungen folgten erst viel später. Der Irrtum kam schließlich nach mehr als drei Wochen rein praktischer Arbeit mit den Studenten zu Tage. Dieser Zufall verhalf jedoch zu der Überzeugung, daß eine einführende theoretische Erklärung der Bildung der einzelnen deutschen Laute gar nicht so notwendig ist, wie man anzunehmen geneigt ist. Nur in solchen Fällen, wo das musikalische Gehör der Studenten ganz und gar versagt, muß eine eingehende Beschreibung nachhelfen. Bei einigen Studenten der Gruppe war dies der Fall. Hier hat dann der "Atlas deutscher Laute" von Hans-Heinrich Wängler als Illustration der Artikulationsbasis besonders gute Dienste geleistet.

Die Adjustierung der Artikulationsbasis bei den Studenten der besprochenen Gruppe wurde, wie schon gesagt, mit dem Einüben der richtigen Lautungsformen der deutschen Vokale begonnen. Die Studenten hatten bisher die deutschen Vokallaute folgendermaßen dem ihnen geläufigen polnischen Vokalsystem zugeordnet: deutsch $\mathrm{a}: \mathrm{a}=\mathrm{a}$, deutsch $\mathrm{o}:, \supset=0$, deutsch $\mathrm{u}:, \mathrm{u}=\mathrm{u}$, deutsch $\varepsilon:, \epsilon$, e:,ə,, , $=\epsilon$; deutsch i:, I, y:,y=i. Sie besaßen also in ihrem System nur fünf Vokallaute, mit denen sie in dem von ihnen gesprochenen Deutsch 16 verschiedene Laute ersetzten (von den fünf Vokalen sind es auch nur drei, die sich in ihrer Artikulationsweise fast ganz mit den entsprechenden Lauten im Deutschen dekken). Von den 16 deutschen Vokallauten sind 15 Phoneme (sie besitzen im Deutschen wortunterscheidende Funktion) und wenn diese nun einer Anzahl von nur fünf Phonemen zugeordnet werden, ist es klar, daß das deutsche Phonemsystem auf eine höchst unzulässige Weise verletzt wird.

Aus der oben angeführten Transponierungsweise geht hervor, daß den Studenten die Artikulationsbasis aller deutscher langer Vokale sowie mit drei Ausnahmen auch die der kurzen Vokale nicht geläufig war und von ihnen erst beherrscht werden mußte. Im gegebenen Fall wurde mit dem Einüben der langen Vokallaute e und o begonnen.

Von den 25 deutschen Konsonantenlauten, von denen 22 Phoneme sind, wurden 21 annähernd oder sogar ganz richtig durch die ihnen im polnischen entsprechenden Konsonanten realisiert. Von den Phonemen 
wurde das (h) als ach-Laut realisiert und fiel so mit diesem zusammen. Von den Varianten fielen dagegen der ich- Laut und der präpalatale Nasal $\mathfrak{y}$, aus, sie wurden durchgehend als ach-Laut und postpalataler Nasal wiedergegeben. Von den $r$-Varianten kam nur das apikale $r$ zum Ausdruck, und zwar mit viel zu starker Vibration. Außerdem fehlte noch die Aspiration bei den stimmlosen Verschlußlauten. Angesichts dieser viel geringeren Interferenz des polnischen Konsonantensystems auf das von den Studenten gesprochene Deutsch, wurden in den ·̈̈bungen nur die Fälle berücksichtigt, die oben genannt wurden.

Beim praktischen Einüben der einzelnen Laute wurde methodisch wie folgt vorgegangen: Ein Einzellaut, der einzuüben war, wurde ohne jede vorausgehende theoretische Einleitung deutlich vom Lektor viele Male gesprochen, die Studenten hörten und sahen $\mathrm{zu}$ und versuchten dann, ihre Artikulationswerkzeuge so einzustellen (besonders die Lippen), daß eine möglichst genaue Reproduktion des gehörten Lautes zustande kommen konnte, ohne jedoch den Laut hörbar auszusprechen.

Auf eine derartige stumme Übung, die hauptsächlich ein Üben des Gehörs zum Ziel hatte, folgte dann ein Mit- und Nachsprechen des Lautes mit höchstens halber Stimmstärke und das im Chor. Der Lektor gab hier zuerst das Muster laut und deutlich an und sprach es auch gleichzeitig mit den Studenten nach. Die Stimme des Lektors war beim Nachsprechen die stärkste und diente gewissermaßen als Stütze für die Versuche der Studenten. Übungen dieser Art sind deshalb zu empfehlen, weil sie die Studenten nicht einschüchtern und ihnen Zeit geben, eine gewisse Sicherheit in der Bildung der Laute zu gewinnen. Es entsteht hier eine ähnliche Situation wie beim Einüben eines Gesangstückes. Der Lektor spielt in diesem Fall die Rolle eines begleitenden Musikinstruments.

Die nächste Etappe der Übung war ein Vorsprechen des Lektors und ein gemeinsames und individuelles Nachsprechen der Studenten, aber diesmal schon ohne Mithilfe des „Instrumentes", d.i. des Lektors. Schon das gemeinsame Wiederholen ermöglichte bei der verhältnismäßig nicht zahlreichen Gruppe das Heraushören von noch fehlerhaften Bildungen bei einzelnen Studenten. Diese wurden dann beim individuellen Nachsprechen durch entsprechend längeres Üben beseitigt.

Nachdem alle Studenten auf die oben beschriebene Weise gelernt hatten, die entsprechende Artikulationsstelle $\mathrm{zu}$ finden, und den gehörten Laut möglichst genau wiederzugeben, erfolgte eine in flottem Tempo gehaltene Übung der einzelnen Laute, indem der Lektor das Modell vorsprach und die Studenten im Chor und individuell nachsprachen, bis eine gewisse Automatisierung der Artikulationsbewegungen zu beobachten war. Danach erschien der geübte Laut im Anlaut und später im In- 
und Auslaut eines Wortes, das sofort in beschleunigtem Tempo durch Vorund Nachsprechen geübt wurde. Es wurde hier besonders darauf geachtet, daß in dem Modellwort nicht noch andere Laute auftraten, die Schwierigkeiten in der Artikulation bereiteten. Das beim Üben eingehaltene schnelle Tempo spielte hier eine sehr wichtige Rolle, denn sie mechanisierte die neuerlernten Artikulationsbewegungen und schloß gleichzeitig ein reflexives Artikulieren aus. Die Studenten waren anfangs durch dieses ungewohnte Tempo etwas verwirrt, aber sie gewöhnten sich dann bald daran und empfanden es als ganz normal.

Nach dem Einüben der einzelnen Laute als solche und in Einzelwörtern (in verschiedenen Stellungen im Wort) durch Hören und möglichst konsequente Wiedergabe, bis eine gewisse Mechanisierung der Artikulationsbewegungen erreicht war, wurden die Laute dann in oppositionell zusammengestellten Wortpaaren im Chor oder einzeln von den Studenten geübt. Es handelte sich vor allem um die wichtigsten distinktiven Oppositionen der deutschen Laute wie: gerundet/ungerundet, stimmhaft/stimmlos, lang/kurz, offen/geschlossen u.a. Hier mag sofort Einspruch erhoben werden und die Tatsache, daß in den Übungen oppositionelle Wortpaare nicht gleich zu Beginn, sondern erst in der dritten Etappe zur Anwendung kamen, als ein schwerwiegender methodischer Fehler bezeichnet werden. Beim Lehren einer Sprache von Grundstufe ab wäre das auch ein durchaus begründeter Vorwurf, aber hier im beschriebenen Fall, wo es sich um eine Adjustierung handelte, wurde bewußt umgekehrt vorgegangen. Es hatte sich nämlich gleich zu Beginn der Übungen gezeigt, daß das Bilden von neuen Lauten ziemlich lange ungemein große Schwierigkeiten physiologischer Art bereitete. Deshalb auch erschien es unmöglich, gleich zwei schwierige Laute auf einmal und diese noch dazu in Wörtern aussprechen zu lassen. Wenn eine oppositionelle Zusammenstellung auch hilft, den Unterschied zwischen den Lauten leichter $\mathrm{zu}$ erfassen und einzuprägen, so war diese Erleichterung doch nicht proportional zu den oben genannten Schwierigkeiten, die bei einer erheblichen Anzahl der Studenten auftraten, und somit ist wohl die erst spätere Anwendung von derartigen Übungen gerechtfertigt. Einzelne, sowie oppositionell zusammengestellte Beispiele für die deutschen Laute fanden sich zur Genüge in dem Übungsbuch von K.-L. Harth: „Deutsche Sprechübungen", Weimar 1961, das auch den Studenten zur Verfügung stand.

Während der phonetischen Übungen wurde immer darauf geachtet, daß das Schriftbild der Modellbeispiele nicht sofort in Erscheinung trat, sondern erst nach der Einprägung des Lautbildes. Die Wichtigkeit dieses Vorgehens ist allgemein bekannt und muß hier nicht besonders besprochen werden. 
In der ersten Zeit der Übungen wurde den recht lerneifrigen Studenten ausdrücklich befohlen, außerhalb der vom Lektor geführten Stunden keine phonetischen Übungen zu machen weil die dabei mögliche Einprägung falscher Formen auf jeden Fall vermieden werden sollte. Phonetische Übungen kamen erst dann als Hausaufgabe in Frage, als die Möglichkeit der Einprägung falscher, interferierender Formen weitgehend ausgeschlossen war, d.h. erst nachdem die gegebenen Laute genügend auf die oben beschriebene Weise geübt worden waren. Erst später, ungefähr nach drei bis vier Wochen Arbeit, durften die vorher in der Gruppe geübten Fragmente aus dem Handbuch von Harth zu Hause wiederholt werden. Nach Beendigung der Arbeit am Aufbau der Artikulationsbasis der wichtigsten sich vom polnischen System grundsätzlich unterscheidenden Laute beruhte die phonetische Hausarbeit der Studenten darauf, daß sie einen kurzen, einer Zeitschrift oder einem Buch entnommenen Text zum Vorlesen vorbereiteten. Beim Vortragen des Textes wurde nur die phonetische Realisation kritisch gewertet.

Nach einer ungefähr zweimonatigen, intensiven Arbeit an der Adjustierung der deutschen Artikulationsbasis der Studenten konnte festgestellt werden, daß sie im allgemeinen imstande waren, alle Laute des deutschen phonetischen Systems sowohl als Einzellaute als auch im Lautkontinuum ohne Mühe relativ richtig auszusprechen. Das bedeutete aber keineswegs, daß damit die alte, eingeprägte fehlerhafte Artikulationsweise beseitigt und vergessen war, sie existierte weiterhin im Unterbewußtsein der Studenten, und als Jahre hindurch eingeprägte Form war sie viel stärker als die neuerlernte. Und hier begann die eigentlich größte Schwierigkeit in der Arbeit, Sobald sich die Studenten nur mit dem Inhaltsplan der Sprache zu beschäftigen begannen, gebrauchten sie völlig unbewußt die alten transponierten phonetischen Formen. Um dieser höchst unerwünschten Erscheinung Herr zu werden, mußte unbedingt etwas unternommein werden, was eine weitere Einprägung der neuerlernten Formen und das Verdrängen der alten förderte. Somit wurden von dieser Zeit ab sämtliche Aussagen der Studenten in den Übungsstunden nicht nur hinsichtlich des Inhaltsplanes sondern auch des Ausdruckplanes und besonders der phonetischen Seite mit, man kann fast sagen, eiserner Konsequenz kontrolliert und wo nötig, korrigiert. Um die Studenten in der ersten Zeit im Inhaltsplan zu entlasten, kamen als Übungsmaterial fertige Monologe, Dialoge u.a. zur Anwendung. Das Material wurde meist neuzeitlichen deutschen Bühnenstücken entnommen und mußte zum größten Teil von Studenten auswendig beherrscht werden. Das Hauptziel dieser ·Übungen war zwar die Bekämpfung einer anderen so hartnäckigen wie unzulässigen Angewohnheit, die die Studenten aus der Schule mitgebracht hatten, und zwar der Ge- 
wohnheit des reflexiven Sprechens (statt Synthese, Analyse), das durch das Einprägen von Metasprache ohne vorhergehendes Beherrschen der Sprache bewirkt wird, und dessen Beseitigung bei Erwachsenen nur zu oft mißlingt, aber diese Übungen erwiesen sich auch für phonetische Zwecke als sehr nützlich. Im zweiten Semester kamen dann zu den genannten Übungen noch freie Gespräche und Erzählen, den Stoff lieferten gemeinsam besuchte deutsche Filme (in Originalversion) oder Werke der modernen deutschen Literatur.

Ungefähr nach 8 Wochen intensiver · Übung, wurde mit der Gruppe eine Tonbandaufnahme gemacht. Es war eine Aufnahme, die eigentlich ganz zufällig zustande kam, und leider in qualitativer Hinsicht viel zu wünschen übrigließ, denn der Raum, in dem sie gemacht wurde, besaß absolut nicht die akustischen Bedingungen, die für Tonbandaufnahmen notwendig sind, es störte vor allem ein viel zu großer Widerhall. Zur Aufnahme kamen zum Vorlesen vorbereitete Texte. Obwohl die Aufnahme eigentlich nur zur Belebung der ·Übungsstunde dienen sollte, gab sie unwillkürlich die Möglichkeit, die bisher erzielten Ergebnisse in der Adjustierung der Artikulation einmal eingehender zu prüfen. Es mußte leider zugegeben werden, daß das Ergebnis der zweimonatigen mühevollen Arbeit recht bescheiden war. Es war zwar schon gelungen, die Aussprache der einzelnen Laute weitgehend $\mathrm{zu}$ verbessern, aber dadurch erhält ein Lautkontinuum der deutschen Sprache im Munde der Studenten noch keineswegs ein wenigstens relativ deutsches Gepräge. Eine weitgehende Interferenz der verschiedenen Systeme des Ausdrucksplanes der polnischen Sprache trat immer noch deutlich hervor. Es interferierten vor allem zwei Faktoren, von denen man in unseren Lehrbüchern meist gar nicht spricht, die aber, wie es sich im beschriebenen Fall zeigte, eine große Rolle spielen.

Es handelte sich erstens um die Interferenz der Art der Assimilation von Lauteigenschaften bei der Koartikulation von größeren Sprecheinheiten im Polnischen, und zweitens um den Einfluß des polnischen Wortakzentes auf die Realisation von deutschen Endsilben. Wie sich die beiden Faktoren auf die Aussprache des Deutschen auswirken, soll hier kurz erklärt werden, denn man gewinnt leider den Eindruck, daß viele Fachkollegen diese beiden Probleme sehr unterschätzen oder auch erst gar nicht in Betracht ziehen.

Im Falle der Assimilation von Lauteigenschaften handelt es sich um die Art der Assimilation von Stimmhaftigkeit und Stimmlosigkeit konsonantischer Laute im An- und Auslaut von Silben und Wörtern bei zusammenhängendem Sprechen. Es besteht in dieser Beziehung ein grundsätzlicher Unterschied zwischen dem Polnischen und dem Deutschen. Im Polnischen haben wir es im Falle einer Aufeinanderfolge von 
auslautendem stimmhaftem Konsonant und anlautendem (sowohl Silbenauslaut als auch Wortauslaut) stimmlosem Konsonant oder umgekehrt, mit einer Assimilation der Stimmlosigkeit oder Stimmhaftigkeit der Laute zu tun, und zwar zu Gunsten des anlautenden, sich zweifellos in stärkerer Position befindenden Lautes, z.B. podpis (potpis), podbój (podbuj), oder przez granicę (przez granice), przez płot (przes płot) u.a. Abhängig von der Qualität des Lautes im Silben- oder Wortanlaut wird also der vorhergehende auslautende Konsonant stimmhaft oder stimmlos ausgesprochen. Im Deutschen werden aber auslautende Konsonanten sowohl im Silben- als auch im Wortauslaut in der Regel stimmlos realisiert, ganz gleich, ob im folgenden Anlaut ein stimmhafter oder stimmloser Laut folgt. Diese Stimmlosigkeit des Auslautes kann in der Koartikulation sogar in manchen Fällen auf den folgenden anlautenden Konsonanten übergreifen, z.B. auf dem (aoftem), auf die (aofti) u.a. Ein entgegengesetzter Proze $\beta$ ist dagegen im Deutschen nicht möglich. Beim Zusammentreffen von Lauten, deren einziges, sie voneinander unterscheidendes Merkmal eben Stimmhaftigkeit oder Stimmlosigkeit ist, kommt es in der besprochenen Situation zum Zusammenfall der Laute und es wird nur ein stimmloser Laut ausgesprochen, z.B. und das (untas), hast dich (hastiç), aufwachen (aofaxen) u.a. Wenn es nun bei einem Deutsch sprechenden Polen zur Interferenz der muttersprachlichen Assimilationsart kommt, wird ein wichtiges deutsches Lautgesetz verletzt. Abgesehen von dessen phonologischer Relevanz (grenzsignalisierende Funktion), wird hier die Reinheit der Aussprache des Deutschen weitgehend beeinträchtigt, denn diese Interferenz hinterläßt einen unverkennbaren „fremden Akzent". Sie gehört zu denen, die am schwierigsten zu bekämpfen sind.

Die zweite obengenannte Interferenzerscheinung hängt mit dem verschiedenartigen Wortakzent in der polnischen und der deutschen Sprache zusammen. Im Polnischen liegt der Wortakżent grundsätzlich auf der vorletzten Silbe des Wortes, er wird durch ein Sprechrhythmisches Bedürfnis bestimmt und deshalb auch als rhythmischer Akzent bezeichnet (er erfüllt phonologisch eine dilimitative Funktion). Im Deutschen liegt der Akzent dagegen auf der Stammsilbe oder einem noch wichtigeren Teil des Wortes und erfüllt so eine logische Aufgabe. Dieser Akzent ist etymologisch bestimmt.

Dieser Unterschied in der Art der Akzentuierung zieht eine grundverschiedene Realisation der Silbenträger, d. i. der Vokale nach sich. Im Polnischen, wo wir es mit einem in gewissem Sinne wandernden Akzent zu tun haben (Pólak, Poláka, Polakówi), also wo er sowohl die Hauptsilbe als auch verschiedene Nebensilben treffen kann, werden alle Vokale des Wortes, auch der der Endsilbe, verhältnismäßig voll reali- 
siert. Im Deutschen aber, wo ständig nur eine Silbe der Akzentträger ist, wird nur der Vokal dieser Silbe voll und deutlich realisiert, die Vokale nichtakzentuierter Silben werden dagegen artikulatorisch stark vernachlässigt. Ein Produkt dieses Prozesses ist der deutsche Endsilbenvokal (ə), der nur noch ein farbloser e-ähnlicher Laut ist, und bei zusammenhängendem Sprechen oft gerade nur noch angedeutet wird oder sogar ganz ausfällt. Eine Bestätigung dieser Beobachtung finden wir in den interessanten Ausführungen über die Realisation des Endsilbenvokals (ə) im Deutschen von G. Meinhold in seinem Beitrag „Formen und Bedingungen der Realisation des Endsilben-e" — Biuletyn Fonograficzny V, 1962.

Die Interferenz der polnischen Akzentuierungsweise wirkt sich im Deutschen derartig aus, daß eine starke Neigung zur vollen Realisation unakzentuierter Silben und besonders des Endsilbenvokals (ə) auftritt. Dieser Vokal wird auch in gedeckten Endsilben als $(\varepsilon)$ realisiert, der Stammsilbenvokal wird dagegen schwächer artikuliert, als es im Deutschen üblich ist. Die fehlerhafte Realisierung des Endsilbenvokals (ə) wird dann außerdem noch durch das Schriftbild unterstützt, das auf keine Weise vermuten läßt, mit welchem Intensitätsgrad der Endsilbenvokal realisiert werden soll und welche Qualität er besitzen muß. Wenn diese Interferenzerscheinung nicht rechtzeitig beseitigt wird, kommt es zu einer unangenehmen Störung des Rhythmus des Gesprochenen. Um dieser Interferenz von vorne herein zu begegnen, ist es zweckmäßig, den Lernenden immer erst das Lautbild des neu zu beherrschenden Materials einzuprägen. Es muß dabei der oft $\mathrm{zu}$ beobachtende Fehler, daß beim Vorsprechen speziell deutlich artikuliert und das Endsilben-e unnötig hervorgehoben wird, unbedingt vermieden werden.

Im beschriebenen Fall hatten sich die Studenten die überbetonte, offene Form des Endsilben-e eingeprägt. Nach einigen Versuchen konnte festgestellt werden, daß dieser Fehler am besten $\mathrm{zu}$ beseitigen war, indem jedes gedeckte Endsilben-e in den auswendig vorgetragenen -Übungstexten reduziert wurde. Es war notwendig, von einer Extreme in die andere $\mathrm{zu}$ fallen, um der richtigen Realisierungsform nahe zu kommen. Ähnlich wie bei der Interferenz der Assimilationsart ist es im Falle eines Adjustierungsversuches hier viel schwieriger als bei den Einzellauten, ein befriedigendes Resultat zu erzielen.

Die Adjustierungsarbeit wiar mit der Aufnahme der Bekämpfung der beiden oben beschriebenen Fälle auf drei größere Interferenzprobleme (die Interferenz des polnischen Lautsystems, der Form der Assimilation von Lauteigenschaften in der Koartikulation und der Art der Realisierung der Endsilben) gerichtet, deren Beseitigung zu einem großen Teil innerhalb eines Studienjahres gelungen ist. 
Kurz vor Ende des zweiten Semesters kam eine zweite Tonbandaufnahme zustande ${ }^{1}$. Sie war ebenso zufällig gemacht worden, wie die erste und nur einem äußerst glücklichen Zufall ist es zu verdanken, daß sie sozusagen als Gegenstück zu der ersten Aufnahme auf die zweite Spur desselben Bandes kam. Den aufgenommenen Stoff bildete diesmal eine freie Inhaltswiedergabe eines vorher gemeinsam besuchten deutschen Filmes.

Unwillkürlich ergab sich durch die Gegenüberstellung der beiden Tonbandaufnahmen die Möglichkeit, das Ergebnis der durchgeführten Adjustierungsarbeit genauer $\mathrm{zu}$ untersuchen. Trotzdem die erste Tonbandaufnahme nicht mehr den Stand vor der Aufnahme der Arbeit fixiert hatte, so war der Unterschied doch immer noch weitgehend. Es erwies sich vor allem, daß die Bildung der Vokale bei allen Studenten keine Schwierigkeiten mehr bereitete und auch schon weitgehend mechanisiert war, so daß die früher durchwegs auftretende interferierende Form schon als verdrängt angesehen werden konnte. Schwankungen bei der relativ richtigen Realisation der Vokale traten ab und zu hinsichtlich der Quantität der Laute auf, und zwar in solchen Wörtern, die zweifellos zum Grundwortschatz der deutschen Sprache gehören und besonders häufig auftreten, sie kommen schon im ersten Jahr des Sprachunterrichts zur Anwendung und sind durch häufigen Gebrauch eingeprägt. Es handelt sieh um solche Wörter wie: haben, fragen, schlafen, liegen, kam (das Wort „kam" wurde sogar oft in schriftlichen Arbeiten mit Doppel-m geschrieben). Die angeführten Beispiele sowie andere dieser Art wurden lange Zeit hindurch immer mit kurzem Stammsilbenvokal realisiert, und trotzdem ihrer Korrektur viel Aufmerksamkeit geschenkt worden war, kam es auch am Ende des zweiten Semesters noch vor, daß z. B. „haben" mit kurzem a realisiert wurde.

In einigen Fällen bereitete die Realisierung der Lautgruppe e:+r im In- und Auslaut trotz intensiver individueller Übungen noch erhebliche Schwierigkeiten, das (e:) wurde immer wieder $\mathrm{zu}$ offen und $\mathrm{zu}$ kurz ausgesprochen. Ein besonders hartnäckiger Fall lag in dem Wort „mehr" vor, das in den Übungen oft auftrat, und zwar weil die Studenten sich statt der deutschen Wendung „nicht mehr" eine wörtliche Übersetzung des polnischen ,już nie" eingeprägt hatten. Sowohl das Ankämpfen gegen die falsche Wendung als auch gegen das zu offene (e:) brachte bis zum Schluß des zweiten Semesters nur einen Teilerfolg und hätte leider noch weiter anhalten müssen. Andere Fehler bei der Realisierung der deutschen Vokallaute, die an Hand der zweiten Tonbandaufnahme

${ }^{1}$ Das Magnettonband mit beiden Aufnahmen befindet sich in der Bibliothek des Lehrstuhls für Germ. Sprachen der UAM in Poznań. 
noch (nachgewiesen werden können, sind als ein gelegentliches „Ausrutschen" zu bezeichnen und dem emotionellen Zustand der Sprecher zuzuschreiben, der durch das ungewohnte Sprechen vor dem Mikrophon hervorgerufen wurde.

Hinsichtlich der deutschen Konsonanten machten die Laute (h) und (ç) nur noch einigen Studenten erheblichere Schwierigkeiten, d. h. es interferierte bei ihnen noch ab und $\mathrm{zu}(\mathrm{x})$. Ein weiteres Üben des Nasals ( $)$ erwies sich dagegen bei fast allen Studenten der Gruppe als notwendig, denn er wurde viel zu oft als (ng) realisiert. Eine Redaktion der Vibration der (r) im In- und Auslaut war dagenen schon weitgehend gelungen (in zwei Fällen durch die Umstellung auf Zäpfchen-r).

Gänzlich unberührt von der Adjustierung blieb vorläufig das Problem der aspirierten Aussprache der deutschen Verschlußlaute sowie des harten Vokaleinsatzes.

Hinsichtlich der Interferenz der Art der Assimilation von Lauteigenschaften wurde ebenfalls beim Vergleich der beiden Tonbandaufnahmen eine merkliche Beseitigung der fehlerhaften Formen beobachtet, aber hier schien eine weitere konsequente Kontrolle und in Einzelfällen sogar das Einschieben von speziellen Übungen unbedingt notwendig, denn der Proze $\beta$ der Festigung der richtigen Realisierung konnte noch nicht als beendet angesehen werden.

Die Praxis hat bewiesen, daß man es hier mit einem der Interferenzfälle zu tun hat, die am schwierigsten zu beseitigen sind. In der besprochenen Gruppe wurden eigentlich sämtliche, in den praktischen Übungen zur Anwendung kommenden Texte (besonders die auswendig vorbereiteten Fragmente aus Theaterstücken) gleichzeitig als Übungen für eine richtige Realisierung der im Deutschen üblichen Assimilation ausgenützt und oft war es notwendig, schwierige Stellen (z.B. „... das Bein, das Bett, das Brot ...", „... das ist der Mann ..." u.a.) gerade deswegen viele Male zu wiederholen, bis das Problem im gegebenen Fall beseitigt war. Trotz derartiger außerordentlicher Bemühungen ist es bis zum Schluß des zweiten Semesters noch nicht gelungen, diese immer wieder hervorbrechende Interferenz zu beseitigen. Von den Studenten selbst wurden ebenfalls die diesbezüglichen Übungen als die schwierigsten bezeichnet, und es ist nicht einmal vorgekommen, daß dabei einer oder der andere mit Resignation erklärte, er würde es nie in seinem Leben erlernen, und die besonders viel geübten Beispiele haben bis heute für die Gruppe sprichwörtliche Bedeutung. Aus dem Angeführten geht zweifellos hervor, daß diesem Problem bei einer Adjustierung besonders viel Aufmerksamkeit geschenkt werden sollte, und daß entsprechendes - Übungsmaterial beschafft werden muß, da es daran so gut wie ganz fehlt.

Beim dritten Interferenzproblem, dem bei der Adjustierung viel Auf- 
merksamkeit geschenkt worden war, d.i. dem ·Überbetonen von deutschen Endsilben und dessen Vokal (ə) konnte ebenfalls ein merklicher Erfolg verzeichnet werden, aber auch hier erschien eine weitere Kontrolle unbedingt notwendig, denn auch hier haben wir es mit einem Interferenzfall zu tun, der einmal eingeprägt, immer wieder auftaucht. Eine genaue Beschreibung des Verlaufs der Adjustierung der Artikulation bei einzelnen Studenten ist leider in diesem Fall unmöglich, da die dazu nötigen entsprechenden Tonbandaufnahmen nicht gemacht worden sind. Es kann nur allgemein gesagt werden, daß alle an den Übungen teilnehmenden Studenten imstande waren, die bei der neuen Artikulation auftauchenden Schwierigkeiten $\mathrm{zu}$ überwinden, nur die dafür notwendige Zeit und Anzahl der·Übungen waren verschieden. Daß einigen Studenten verhältnismäßig schnell eine weitgehende Umstellung gelang, ist sicher ihrem großen Nachahmungsvermögen sowie einer guten Gehörschulung zuzuschreiben, es war aber nicht altersbedingt. Der Unterschied zwischen einigen mehr Begabten und dem Rest der Gruppe war zum Schluß des zweiten Semesters keineswegs groß und konnte in Einzelfällen nur von Eingeweihten wahrgenommen werden. Es muß besonders betont werden, daß alle Studenten der Gruppe die Ausspracheübungen außergewöhnlich ernst nahmen und mit lobenswertem Eifer bemüht waren, die diesbezüglichen Aufgaben auf das gewissenhafteste zu erfüllen. Wieviel mühevolle Arbeit außer den Übungen in der Gruppe von einigen noch $\mathrm{zu}$ Haus geleistet wurde, um diese oder jene Form zu erlernen, läßt sich nur ahnen. Die Adjustierung der Artikulation kam letzten Endes bei 9 Studenten weitgehend zustande. Zwei Studenten verließen im Laufe des Jahres das Studium, und der älteste Student der Gruppe konnte bedauernswerterweise von der Adjustierungsarbeit nicht erfaßt werden, da er die Übungen aus bestimmten Gründen äußerst unregelmäßig besuchte. Bei einem der Studenten waren die Übungen in der zweiten Hälfte des ersten Semesters durch einen sechswöchigen Aufenthalt in Deutschland unterbrochen worden. Die Pause in den Übungen hatte hinsichtlich der Phonetik keine nennenswerten Lücken hinterlassen, der Aufenthalt in Deutschland dagegen keine Fortschritte bewirkt.

Wenn man aus der Perspektive der Zeit auf die geleistete Arbeit zurückschaut, so muß zugegeben werden, daß einige methodische Fehler begangen worden sind, die in Zukunft unterbleiben müssen. Erstens war es methodisch falsch, die Adjustierung der Aussprache der Vokale von (e:) und (o:) zu beginnen, denn dadurch wurde das Prinzip der Steigerung der Schwierigkeiten in hohem Grade verletzt. Ein zweiter Fehler war es, das Üben der deutschen kurzen Vokale, die den polnischen artikulatorisch näher stehen, stark zu vernachlässigen. Die Praxis hat gezeigt, 
daß sämtliche Vokale des deutschen Lautsystems im Falle einer Adjustierung der Artikulationsbasis ganz und gar von neuem aufgebaut und bis zu einer Mechanisierung der Artikulationsbewegungen und damit bis zu einer Ausschließung der Interferenzmöglichkeit der in der Muttersprache nahestehenden Laute eingeübt werden. Die Proportion der auf die Einübung der einzelnen Lautformen zu verwendenden Zeit ist noch $\mathrm{zu}$ ermitteln.

Unrichtig war es außerdem, die Aufmerksamkeit auf Assimilation und Reduktion erst nach zwei Monaten der Arbeit zu richten. Diese beiden Probleme sind unbedingt gleich von Anfang an zu berücksichtigen, damit das weitere Einprägen dieser hartnäckigen Interferenzfälle ausgeschlossen wird.

Einige Fachkollegen wird es vielleicht interessieren, warum bei der Arbeit kein Tonbandgerät zur Anwendung kam. Erstens ist es aus bestimmten Gründen nicht möglich gewesen, ständig ein Gerät zu benutzen und zweitens sei bemerkt, daß die Meinung vertreten wird, daß der Anwendung eines mechanischen Gerätes bei einer Adjustierung der Artikulation keine große Rolle beizumessen ist. Es kann zur Kontrolle der erreichten Resultate bei einzelnen Personen verwendet werden, nicht aber beim Üben der neu zu erlernenden Formen. Das Modell muß unbedingt ein Lektor sprechen, denn die visuelle Seite spielt beim Nachsprechen unbekannter und ungewohnter Laute eine ungewöhnlich große Rolle, und die Übungen sind ohne maschinelle Hilfe viel elastischer, da es dem Lektor jederzeit möglich ist, Unzulänglichkeiten herauszuhören und diese sofort zu beseitigen, oder das Beispiel soviele Male zu wiederholen, wie es gerade die gegebene Situation verlangt, u.dgl.m. Dies alles kann ein Tonbandgerät $\mathrm{nicht}$ und es sind leider Faktoren, die bei derartigen $\cdot$ Übungen unentbehrlich sind.

Es gibt gewiß noch viele andere Probleme auf die der beschriebene Versuch einer Adjustierung der Artikulationsbasis bei Studenten der Germanistik ein Licht geworfen hat, aber es ist hier unmöglich, alle eingehend $\mathrm{zu}$ besprechen. Es ist auch unbedingt notwendig, noch weitere Versuche durchzuführen und die erhaltenen Ergebnisse miteinander $\mathrm{zu}$ vergleichen. Es ist bestimmt $\mathrm{zu}$ früh, schon nach einem Versuch, und dazu noch nach einem unabgeschlossenen ${ }^{2}$, weitgehende, wissenschaftlich wertvolle Schlüsse zu ziehen, aber eines konnte mit diesem Versuch unumstößlich bewiesen werden, und zwar, daß eine Adjustierung der Artikulation bei fehlerhafter Aussprache bei Studenten der Germanistik

${ }^{2}$ Der Versuch, der noch nicht bis zum vollen Erfolg geführt hat und mindestens noch ein Semester dauern müßte, ist inzwischen unabhängig von der Verfasserin des Berichts unterbrochen worden. 
ohne weiteres möglich ist, und diese Tatsache muß schon jetzt ausgenutzt werden, um den großen, in dieser Hinsicht bei uns herrschenden Mißstand zu beseitigen. Es wird hier bestimmt der Einwand erhoben werden, daß es Sache der Oberschule sei, sich um eine gute Aussprache der gelehrten Fremdsprache zu kümmern, weil dort die Sprache von Grundstufe an gelehrt werde, und die Universität habe nur die theoretische Seite der Sprache ins Auge zu fassen. Dieser Standpunkt mag auch einerseits richtig sein, aber andererseits muß doch jemand angesichts der anormalen Situation den Lehrern der Oberschule (die ihre Fachausbildung an der Universität erhalten) ein vollwertiges Arbeitswerkzeug in die Hände geben, damit sie nachher imstande sind, ihren Schülern die nötigen, richtigen praktischen Grundlagen der Sprache zu vermitteln. In absehbarer Zeit würde das Problem der Adjustierung der Artikulation dann aufhören, eins zu sein. 